\title{
Revisiting a study of callosal apraxia: The right hemisphere can imitate the orientation but not the position of the hand
}

\author{
Biljana Petreska ${ }^{\mathrm{a}, *}$, Aude Billard ${ }^{\mathrm{a}}$, Joachim Hermsdörfer ${ }^{\mathrm{b}}$, Georg Goldenberg ${ }^{\mathrm{c}, \mathrm{d}}$ \\ a Learning Algorithms and Systems Laboratory, Ecole Polytechnique Fédérale de Lausanne, Lausanne, Switzerland \\ b Clinical Neuropsychology Research Group (EKN), Klinikum München-Bogenhausen, Munich, Germany \\ c Department of Neuropsychology, Bogenhausen Hospital, Munich, Germany \\ d Department of Neurology, Technical University Munich, Germany
}

\section{A R T I C L E I N F O}

\section{Article history:}

Received 14 July 2009

Received in revised form 9 April 2010

Accepted 22 April 2010

Available online 7 May 2010

\section{Keywords:}

Apraxia

Imitation of meaningless gestures

Lateralization

Hemisphere specialization

Corpus callosum

\begin{abstract}
A B S T R A C T
Callosal disconnection can reveal asymmetrical contributions of the two brain hemispheres to praxis. In this paper, we revisit a study of a patient with callosal disconnection (Goldenberg et al., 2001, Neuropsychologia, 39:1432-1443), who perfectly imitated meaningless gestures when imitation was controlled only by the left hemisphere, but was severely impaired when the right hemisphere was in charge of motor control. We decomposed the gestures into a set of geometric variables that were to be reproduced, such as the orientation of the hand and the position of contact between the hand and the face. Whereas orientation of the hand in extrinsic coordinates was replicated correctly by both hemispheres, only the left hemisphere reproduced correctly the position of contact between the hand and the face. This goaldissociation as well as several partial perseveration errors speak against the hypothesis of a direct route from perception to motor replication of gestures, as interruption of a direct route would probably impair all the features of the gesture. We speculate that incorrect coordination between the reproductions of multiple goals may be the core deficit underlying callosal apraxia.
\end{abstract}

(c) 2010 Elsevier Ltd. All rights reserved.

\section{Introduction}

Apraxia refers to a disorder of the high-level control of voluntary movement, and is described in terms of the absence of certain deficits rather than in terms of its mechanisms, which are still poorly understood (Petreska, Adriani, Blanke, \& Billard, 2007). Specifically, apraxia is defined as "a disorder of skilled movement not caused by weakness, akinesia, deafferentation, abnormal tone or posture, movement disorders such as tremor or chorea, intellectual deterioration, poor comprehension, or uncooperativeness" (Heilman \& Rothi, 1993). Since the apraxia's symptoms exclude elementary sensory and motor deficits, apraxia is particularly appropriate for studying the neural processes underlying specific sensori-motor transformations, such as visuo-motor imitation. Case studies of impaired imitation following apraxia, such as the one revisited here, offer a valuable route to deciphering the neural functions and mechanisms of imitation (De Renzi, Faglioni, \& Sorgato, 1982; Goldenberg \& Hagmann, 1997; Merians et al., 1997; Ochipa, Rothi, \& Heilman, 1994; Tessari, Canessa, Ukmar, \& Rumiati, 2007). In particular, they have revealed important dissociations

\footnotetext{
* Corresponding author at: EPFL-STI-I2S-LASA, Station 9, CH 1015 Lausanne, Switzerland. Tel.: +412169354 64; fax: +41216937850.

E-mail address: biljana.petreska@a3.epfl.ch (B. Petreska).
}

between the imitation of meaningless and meaningful gestures, and elements of the hemispheric specialization of praxis (e.g., left hemisphere dominance); see Petreska et al. (2007) for a review.

This paper revisits a case study of imitation of meaningless gestures by a patient whose brain hemispheres were disconnected by a callosal lesion which included the splenium (Goldenberg, Laimgruber, \& Hermsdörfer, 2001). This seminal study examined the reproduction of hand postures relative to his face (shown in Fig. 1A). ${ }^{1}$ In order to disentangle the contributions of each brain hemisphere, the stimuli to imitate were presented tachistoscopically either in the left or right visual field. In both cases, the patient was requested to imitate alternatively with either the left or right hand (see Fig. 1B). A quantitative analysis of the patient performance revealed that the imitation of hand postures relative to the face was preserved only in the "right visual field-right hand" condition; that is, when both perception and motor execution were processed uniquely by the left hemisphere. This observation suggests that the visuo-motor imitation of meaningless gestures requires key competences located in the left hemisphere (see Fig. 1C).

\footnotetext{
1 The study also examined the imitation of finger postures, which will not be considered here as the underlying neural substrates are different from those associated with the imitation of hand postures (Goldenberg \& Karnath, 2006).
} 
(A)

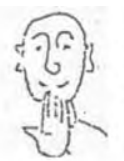

1

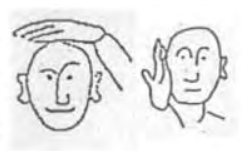

2

3
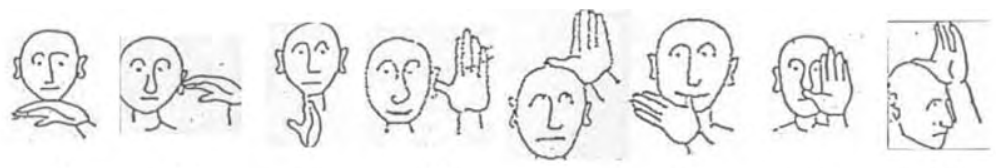

(B)

Stimulus

4

5

6

7

8

$\begin{array}{ll}9 & 10\end{array}$

11
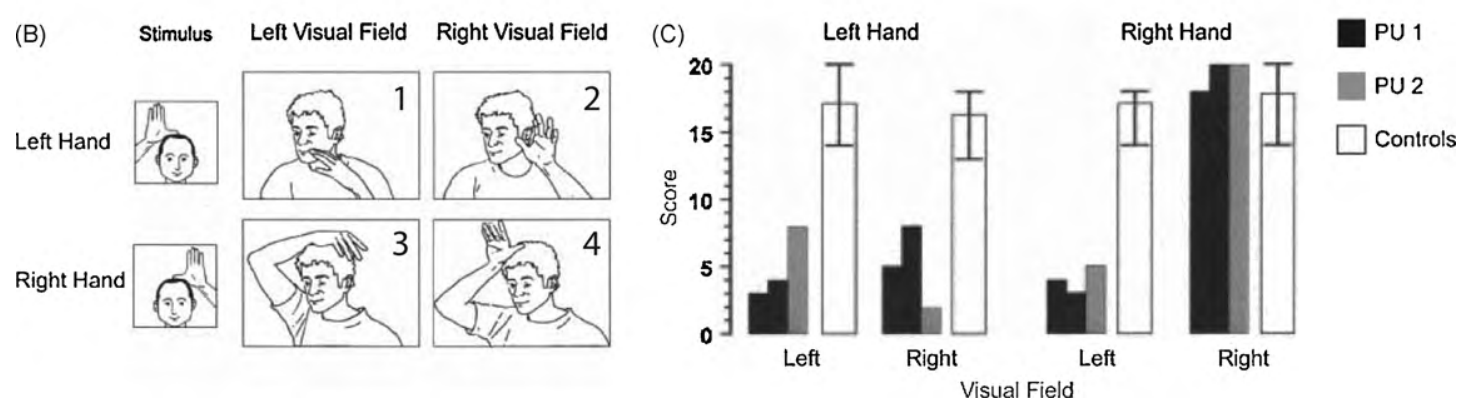

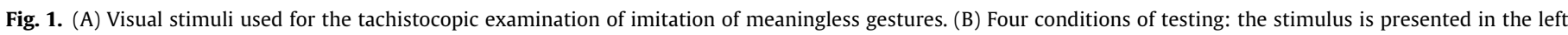

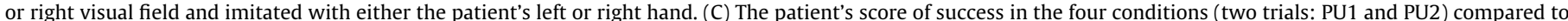

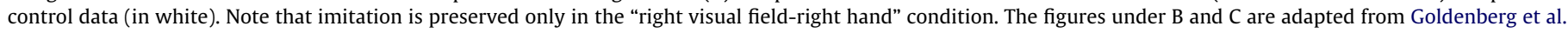
(2001) by permission of Elsevier.

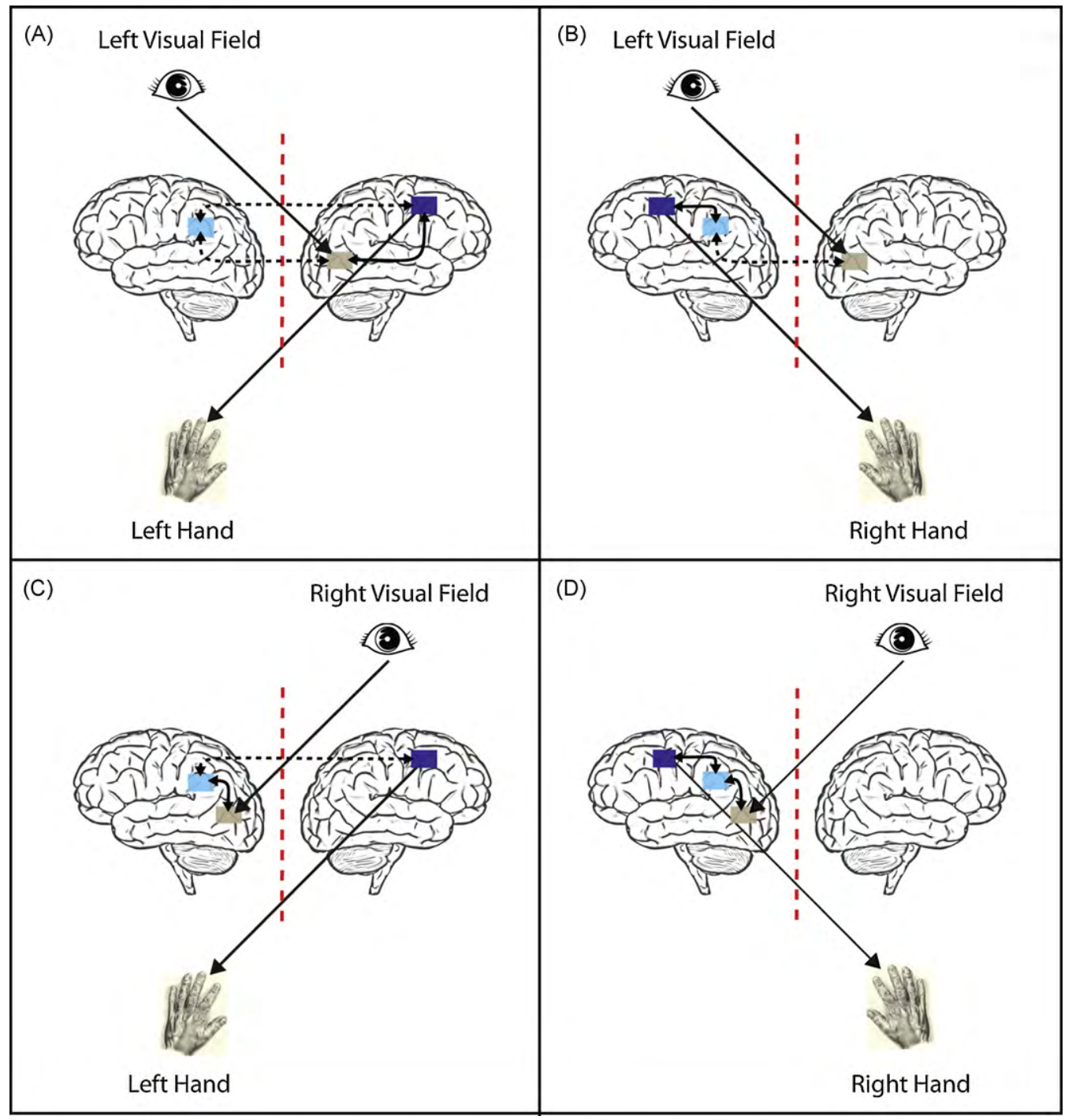

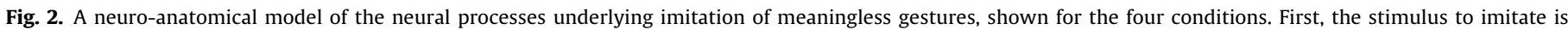

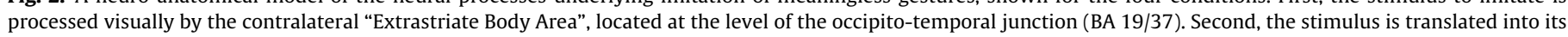

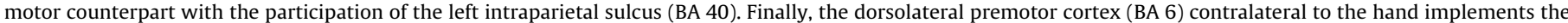
motor component of imitation, possibly through motion attractors. 
In a previous work, we proposed a neuro-anatomical model for the information flow across hemispheres during the imitation of meaningless gestures (Petreska \& Billard, 2006). The model made predictions about the brain areas and neural processes that support this information flow, based on evidence from brain imaging and brain lesion studies (see Fig. 2). According to this model, a gesture demonstrated within one visual hemifield will be first processed visually in the contralateral hemisphere, possibly in the "Extrastriate Body Area" situated at the occipito-temporal junction in Brodmann Area (BA) 19/37, specialized for processing pictures of the human body or body parts (Astafiev, Stanley, Shulman, \& Corbetta, 2004; Downing, Jiang, Shuman, \& Kanwisher, 2001; Peigneux et al., 2000). A further crucial station on the way from visual perception to motor replication of gestures is the left parietal cortex (BA 40, near the intraparietal sulcus), as indicated by PET and fMRI brain neuroimaging studies during the observation or imitation of meaningless gestures (Decety et al., 1997; Hermsdörfer et al., 2001; Mühlau et al., 2005; Peigneux et al., 2004). These functional imaging studies are complemented by lesion studies that also show a left hemisphere dominance for the imitation of meaningless hand gestures (Goldenberg, 1996; Hermsdörfer et al., 1996; Weiss et al., 2001), where the inferior parietal lobe is a common denominator to all observed lesions (Goldenberg \& Karnath, 2006; Haaland, Harrington, \& Knight, 2000; Tessari et al., 2007). Finally, neural activation is consistently observed in a motor brain area contralateral to the imitating hand, namely the dorsolateral premotor cortex (BA 6) (Decety et al., 1997; Hermsdörfer et al., 2001; Mühlau et al., 2005; Peigneux et al., 2004). This brain area may implement the motor command of the hand in the form of a motion attractor which guides imitation by a representation of the desired hand posture (Petreska \& Billard, 2009).

However, the statistical analysis of the patient's errors in Goldenberg et al. (2001) is not sufficient to pinpoint the exact nature of the contribution of the left hemisphere (see Fig. 2A). While we know that there is an indispensable left-lateralized process, we do not know for what this neural process is specialized. To better delineate this process, we re-analyzed the patient's errors by defining a set of objective geometric variables that the brain must reproduce in order to correctly perform the imitation task. Roughly comparable error notation systems have been suggested by Haaland and Flaherty (1984), Rothi, Ochipa, and Heilman (1997), Rothi, Raymer, and Heilman (1997), Haaland et al. (2000), HannaPladdy et al. (2001), and Hanna-Pladdy, Heilman, and Foundas (2001). We hypothesized that a finer analysis of the error pattern for each condition and geometric variable would allow us to: (a) better understand the role that each hemisphere plays in the process of imitation of meaningless gestures, (b) explain the fact that reproduction of only some of the stimuli is impaired (see Fig. 1C) and (c) highlight the elements of the gesture, if any, that are incorrectly reproduced. Finally, we consider the motor response in relation to the visual stimulus so as to find correlations across visual and motor representations that may pinpoint to specific neural deficits at the basis of callosal visuo-imitative apraxia.

\section{Methods}

\subsection{Case report}

The patient, PU, had suffered a spontaneous bleeding from an arterio-venous malformation in the territory of the anterior cerebral artery. The malformation was surgically resected but the operation resulted in destruction of middle and posterior third of the corpus callosum, including the splenium. He was seen 2 years after the operation, at the age of 36. MRI showed destruction of truncus and splenium coroporis callosi with visible preservation of some fibres in the central and parietal portion. On the left side the lesion extended into the posterior thalamus affecting the pulvinar and the nucleus ventrolateralis posterior. However, callosal transfer of information was not completely interrupted, as evidenced by above chance performance on the imitation of meaningless gestures test (see Fig. 1C). For a detailed

\section{Angles: \\ Positions:}

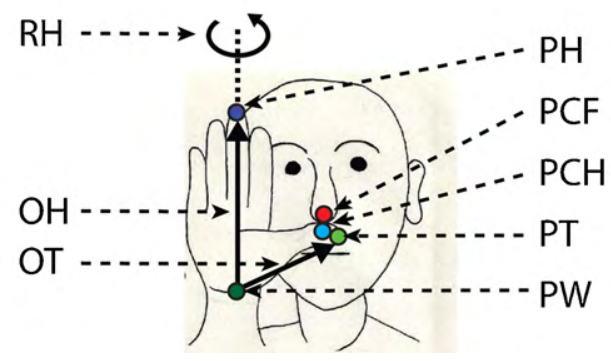

Fig. 3. Geometric variables used for scoring the patient's performance. Two variables are related to the position of contact (PC) between the face (PC-F) and the hand $(\mathrm{PC}-\mathrm{H})$. Three variables are angles related to the posture of the hand (HP): orientation of the hand $\mathrm{OH}$ (relates the positions of the tip of the hand $\mathrm{PH}$ and wrist $\mathrm{PW}$ ), rotation of the hand $\mathrm{RH}$ and orientation of the thumb OT (relates the positions of the tip of the thumb PT and wrist PW).

description of the patient's performance on various neuropsychological tests and MRI images of the lesion see Goldenberg et al. (2001).

\subsection{Procedure}

The subject was seated in front of a computer monitor. At the start of each trial a central fixation point was shown for $1000 \mathrm{~ms}$. Then the stimulus for imitation appeared either in the left or the right visual field. Stimuli subtended a visual angle of $8^{\circ} \times 8^{\circ}$ and their medial edge was $2^{\circ}$ lateral from fixation. Duration of presentation was $150 \mathrm{~ms}$ for controls and $180 \mathrm{~ms}$ for PU who in preliminary trials with $150 \mathrm{~ms}$ ascribed his difficulties to the shortness of presentation and asked for a longer duration of the stimuli. Fixation was controlled by an experimenter standing behind the monitor. Within each block of trials the same hand was examined, while the laterality of stimulus presentation varied randomly.

\subsection{Stimuli}

The stimuli used for the study depict meaningless postures of the hand relative to the face with line drawings (Fig. 1A). A left hand was shown for right-handed imitation and vice versa. Eleven different gestures appeared at least once and a maximum of three times in both the right and the left visual fields.

\subsection{Data analysis}

For fine-grained error analysis we broke down the visual stimuli into five objective geometric variables, which in combination entirely define the stimulus (see Fig. 3). The variables could be classified into two separate measures of how well the stimulus was reproduced:

(a) Geometric variables related to the position of contact between the face and the hand (PC), which include:

(1) PC-F, position of contact on the face.

(2) PC-H, position of contact on the hand.

(b) Geometric variables related to the hand posture per se (HP), which include:

(3) $\mathrm{HP}-\mathrm{OH}$, orientation of the hand in extrinsic space, defined as the vector between the position of the tip of the middle finger and the position of the wrist.

(4) HP-RH, angle of rotation of the hand about the axe of the forearm.

(5) HP-OT, orientation of the thumb in extrinsic space, defined as the vector between the position of the thumb and the position of the wrist. Note that HP-OT is irrelevant for the first half of the stimuli (see Fig. 1A).

PU's performance was scored by computing errors in the partial or complete reproduction of each of the stimuli according to each of the above geometric variables. The imitation of the position of contact was coded as follows. We subdivided the face and hand into parts: (1) nose, mouth, chin, cheek, forehead and ear; and (2) thumb, 4 fingers, palm and back of the hand. A score of 0 was given whenever the patient was not touching with the correct part of the face or hand (e.g., the patient was touching the nose instead of the ear). A score of 1 was given whenever the patient was touching with the correct part of the face or hand but not at the correct location (e.g., with the tip of the thumb instead of its side). Finally, a score of 2 was given when the correct part was touched at the correct location. The imitation of the hand posture was coded as follows. We gave a score of 0 when the angle between the imitated and correct orientations exceeded $45^{\circ}$ (e.g., the hand was horizontal instead of vertical), a score of 1 when this angle was between $30^{\circ}$ and $45^{\circ}$, and a score of 2 when the discrepancy was less than $30^{\circ}$. The examination was video-taped and rated independently by two judges. Interrater reliability was satisfactory $(r>0.9)$. 
Table 1

Mean scores ( \pm standard deviation) given for each condition per geometric variable, imitation goal and two groups of stimuli.

\begin{tabular}{|c|c|c|c|c|c|c|c|c|c|}
\hline \multirow[t]{2}{*}{ Condition } & \multicolumn{9}{|l|}{ Variable } \\
\hline & PC-H & PC-F & HP-RH & HP-OT & $\mathrm{HP}-\mathrm{OH}$ & PC & HP & No thumb(stimuli 1-6) & Thumb(stimuli 7-11) \\
\hline LL & $1.42 \pm 0.82$ & $1.67 \pm 0.54$ & 2 & 2 & $1.97 \pm 0.1$ & $1.55 \pm 0.69$ & $1.99 \pm 0.06$ & $1.93 \pm 0.09$ & $1.67 \pm 0.52$ \\
\hline RL & $1.03 \pm 0.96$ & $1.55 \pm 0.64$ & $1.39 \pm 0.87$ & $1.42 \pm 0.75$ & $1.52 \pm 0.76$ & $1.29 \pm 0.88$ & $1.44 \pm 0.81$ & $1.88 \pm 0.02$ & $0.79 \pm 0.47$ \\
\hline LR & $1.36 \pm 0.88$ & $1.36 \pm 0.88$ & $1.64 \pm 0.77$ & $1.64 \pm 0.77$ & $1.79 \pm 0.46$ & $1.36 \pm 0.9$ & $1.69 \pm 0.7$ & $1.73 \pm 0.15$ & $1.35 \pm 0.32$ \\
\hline $\mathrm{RR}$ & 2 & 2 & 2 & 2 & 2 & 2 & 2 & 2 & 2 \\
\hline All except RR & $1.27 \pm 0.72$ & $1.53 \pm 0.45$ & $1.68 \pm 0.43$ & $1.69 \pm 0.33$ & $1.76 \pm 0.27$ & $1.4 \pm 0.63$ & $1.7 \pm 0.36$ & $1.85 \pm 0.15$ & $1.35 \pm 0.32$ \\
\hline
\end{tabular}

\subsection{Statistical analysis}

A three-factor analysis of variance (ANOVA) with within-subject factors: "condition" (4 levels: LL, LR, RL and RR), "geometric variable" (5 levels: PC-F, PC-H, HP-OH, HP-RH and HP-OT) and "stimulus" (11 levels) was carried out for the patient's score. Two-factor analyses of variance (ANOVA) were conducted to explore interactions. We also performed a new set of similar analysis (including $t$-tests) by grouping the levels of factor "geometric variable" into a new factor "goal" (2 levels, position of contact between the hand and the face PC and the hand posture HP), and those of factor "stimulus" into a new factor "thumb" (2 levels, stimuli 1-6 where the thumb is not shown vs. stimuli 7-11 where the thumb is relevant).

\section{Results}

We first perform a quantitative error analysis in order to identify which of the geometric variables were impaired. In order to understand how apraxia affects these variables, we then turn to a detailed qualitative analysis, by categorizing the errors observed in each condition.

\subsection{Quantitative analysis of the errors}

The three-way analysis of variance on the patients' performance showed that all three factors had significant effects. A main effect of "condition" indicated that the crossed conditions LR and RL (where perception and motor execution were processed by a different hemisphere) were significantly more impaired than the ipsilateral conditions LL and RR $\left(F_{3,120}=26.27, P<0.001\right)$. Note that PU's score in the RR condition was perfect. A main effect of "geometric variable" $\left(F_{4,120}=5.94, P<0.005\right)$ indicated that the position of contact located on the hand $(\mathrm{PC}-\mathrm{H})$ was affected most, followed by the position of contact located on the face (PC-F). A main effect of "stimulus" indicated that not all the imitation gestures were similarly affected $\left(F_{10,120}=12.8, P<0.001\right)$. We found one significant interaction effect between the factors "condition" and "stimulus" $\left(F_{30,120}=6.89, P<0.001\right)$ indicating that the gestures were not equally impaired across conditions.

\subsubsection{Effect of the geometric variable}

Reproduction of all variables was defective but there was a gradation in the severity of impairment (details are given in Table 1). Following this gradation, we grouped the geometric variables into a new factor "goal" with 2 levels: position of contact (PC), and hand posture (HP). For each condition we performed a two-way ANOVA with this new factor and the factor "stimulus". We observed a main effect of "goal" in conditions $\operatorname{LL}\left(F_{1,33}=19.52, P<0.001\right)$ and $\operatorname{LR}\left(F_{1,33}=12.54, P<0.005\right)$ implying that, in the left visual field conditions, imitation of the position of contact was significantly more impaired than imitation of the hand posture (see Fig. 4A).

\subsubsection{Effect of the stimulus}

To explore the interaction between the factors "stimulus" and "condition" we performed one-way ANOVAs for "stimulus" in each condition, which showed that stimuli 8 and 9 were imitated significantly worse than the other stimuli in condition $\operatorname{RL}\left(F_{10,44}=9.43\right.$, $P<0.0001$ ), and stimuli 1 and 10 were imitated significantly worse in condition $\operatorname{LR}\left(F_{10,44}=11.26, P<0.0001\right)$.
We then separated the stimuli into two groups according to whether the face was touched with the thumb (stimuli 7-11) or not (stimuli 1-6). By comparing these two groups with a paired $t$-test we found significant differences in two of the conditions (LL: $T_{53}=2.1, P<0.05$, RL: $T_{53}=6.37, P<0.0001$ and LR: $P>0.05$ ) where the performance was much worse in gestures that involved the thumb (see Table 1). Note that stimuli 2, 4 and 6 were perfectly imitated in all conditions (see Supplementary Material, Fig. 1).

\subsection{Qualitative analysis of the errors}

Here we present a qualitative description of the errors that may help to understand the precise cause underlying the deficit.

\subsubsection{Ipsilateral condition: left visual field-left hand}

All of the errors made by the patient in the left visual field-left hand condition (LL) are related to the position of contact between the hand and the face, whereas the posture of the hand was imitated correctly (shown in Fig. 4C). According to our model illustrated on Fig. 2, these errors speak in favor of a specific role of the left hemisphere for the imitation of the position of contact. The deviations from the correct position of contact are not uniformly distributed around the desired position. Specifically, the directions and amplitudes of the error vectors cover a limited sector of $135^{\circ}$ on the right part of the frontal plane and show a tendency for underreaching (see the last quadrant of Fig. 4C).

\subsubsection{Crossed conditions: right visual field-left hand and left visual field-right hand}

We observed 2 errors similar to those in condition LL(e.g., wrong position of contact with a correct hand posture) only in the right visual field-left hand condition (RL). The most prominent errors were perseverations, where the previous LL stimulus was either totally repeated (RL: 4 instances out of 6, LR: 3 instances out of 4) or only the previous hand posture was combined with an updated position of contact between the hand and the face (RL: 2 instances, LR: 1 instance). In Fig. 4B we give an example of this particular variant of perseveration. ${ }^{2}$ In condition RL there were also 2 "no-idea" errors during the imitation of stimuli 3 and 9, where the patient expressed total ignorance about the stimulus to imitate and did not take any action. In condition LR there were 4 errors characterized by extensive searching, which consisted of a sequence of up to six distinct simple postures that could not be related to any of the presented stimuli ( 3 of these errors were observed when the patient imitated stimulus 10).

\footnotetext{
2 Note that within one block of trials the hand was blocked and the visual field was randomly varied; such that LL and RL stimuli (similarly LR and RR stimuli) were randomly intermingled within one block of trials (see Section 3.2).
} 
(A)

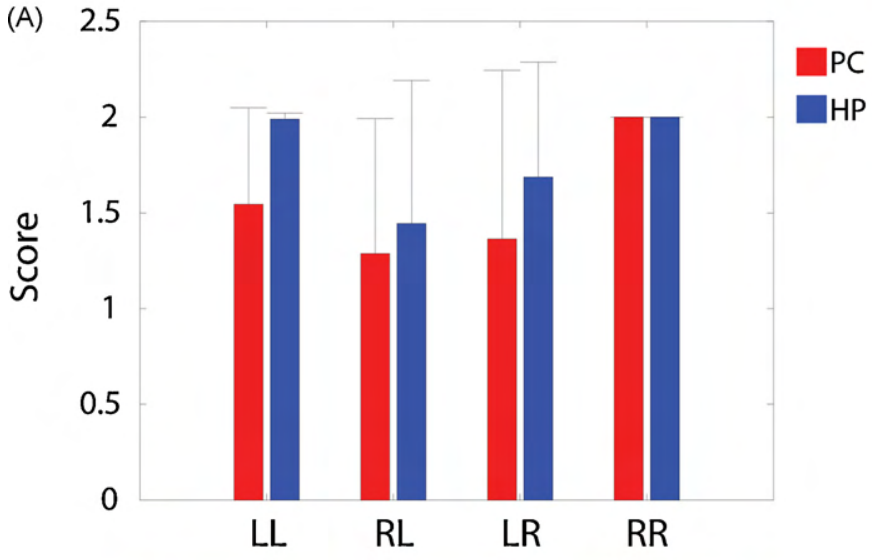

(B)

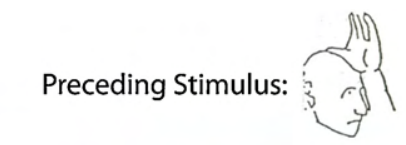

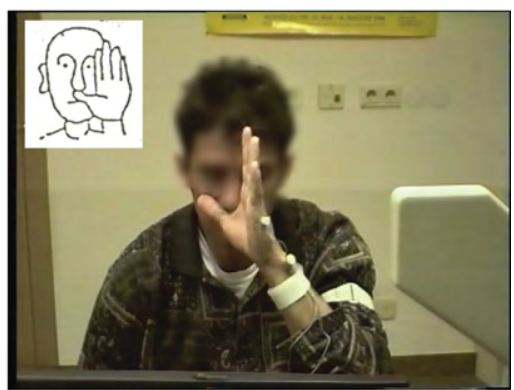

(C)
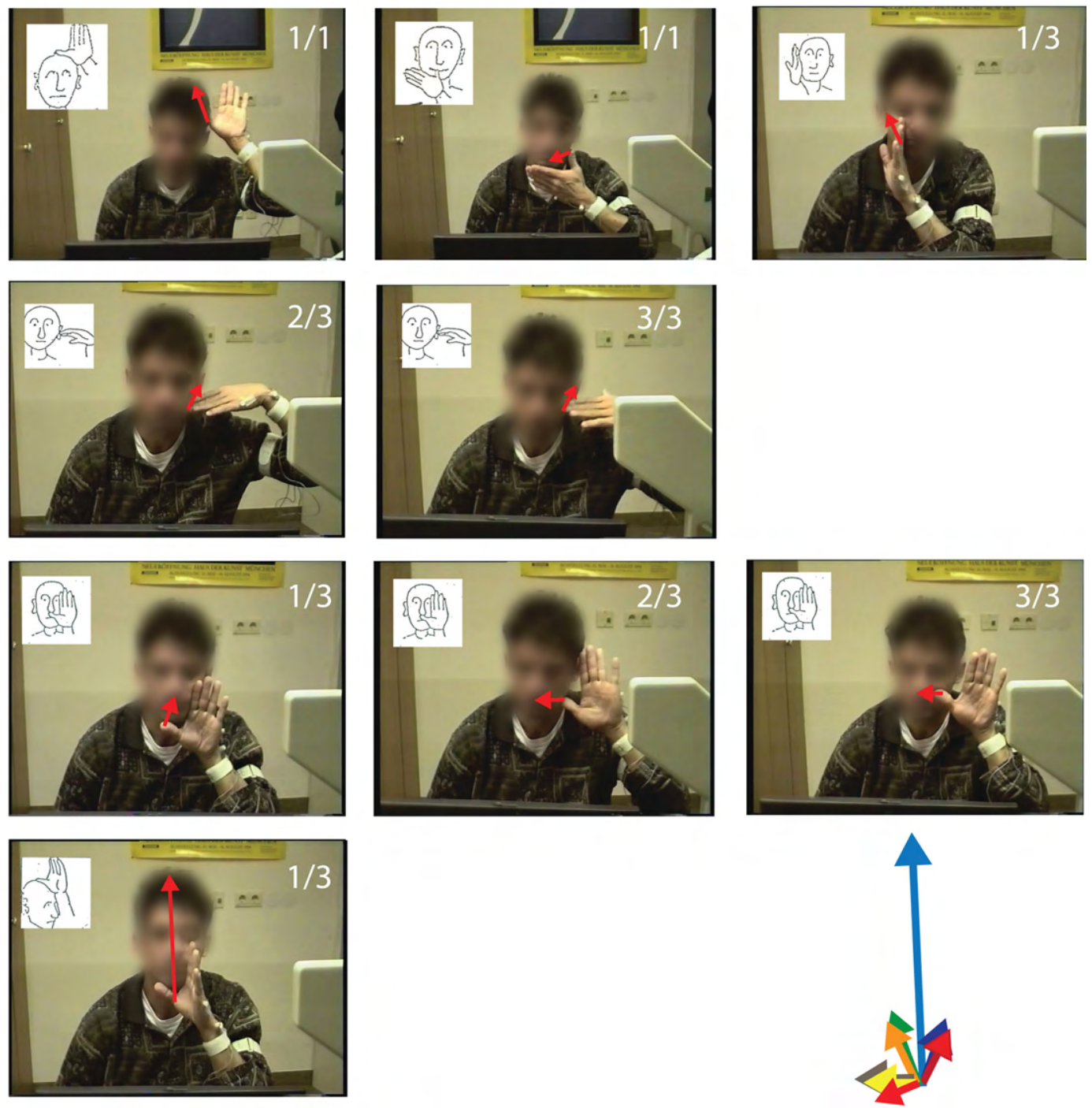

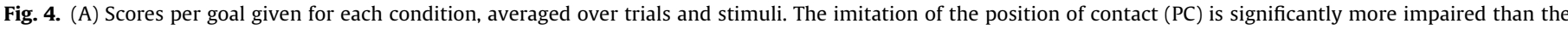

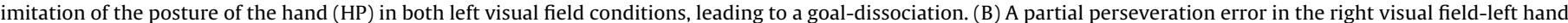

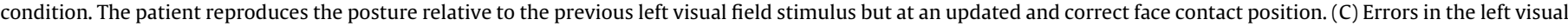

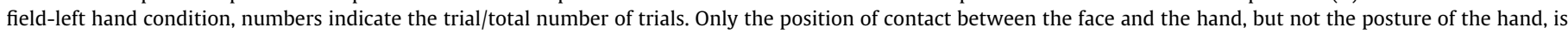
affected. The last quadrant shows superimposed vector deviations from the underreached correct position.

\section{Discussion}

In this paper we re-analyzed the imitation errors of a patient with callosal apraxia presented in Goldenberg et al. (2001). We scored impairments according to the reproduction of five geometric variables that can be related to either the posture or the position of the hand. Our results lead to three observations: (a) reproduction of the position of the hand on the face is significantly more impaired 
Stimulus to imitate
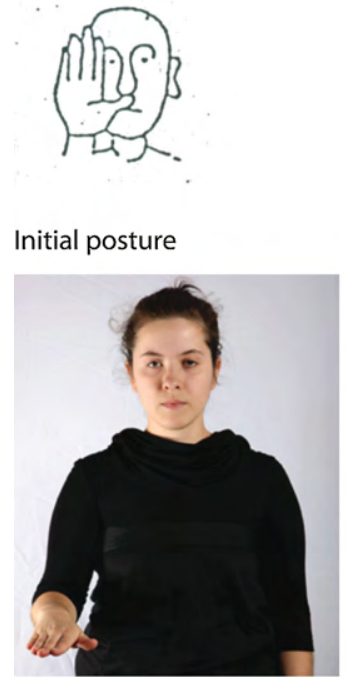

\section{A. Imitation of the hand posture first}
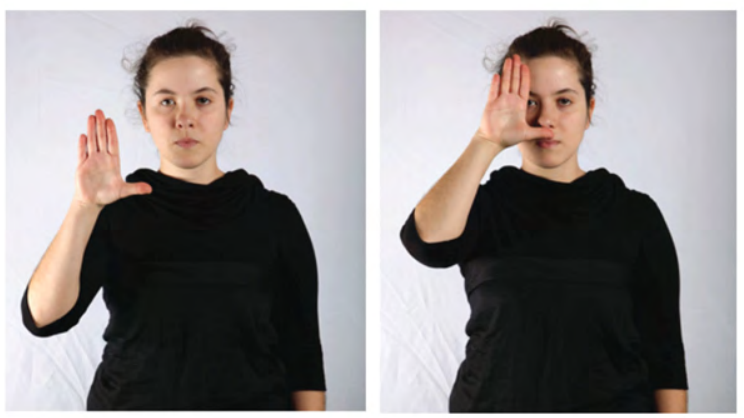

B. Imitation of the position of contact first
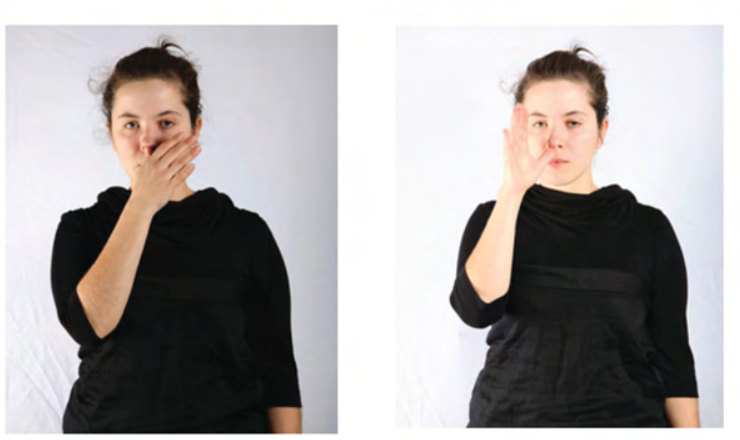

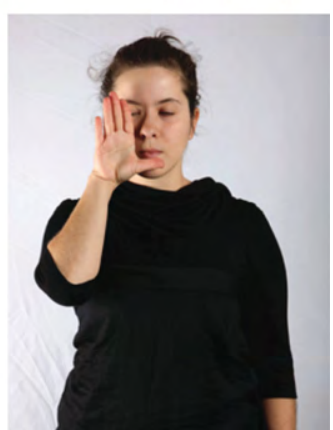

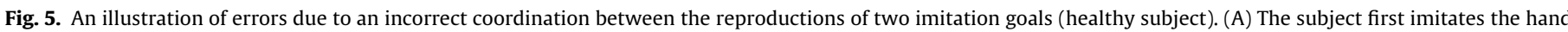

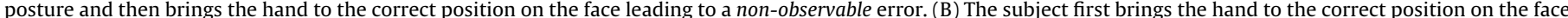

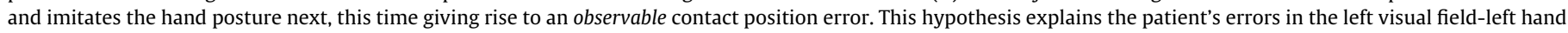
condition and differential performance across trials (see Fig. 4C).

than reproduction of the orientation of the hand relative to the vertical or horizontal direction of extrinsic space; (b) reproduction of the orientation of the hand is entirely preserved in the ipsilateral conditions, i.e., when both visual processing and motor execution recruit the same hemisphere; (c) localization of the thumb on the face is more impaired than that of the other fingers.

When imitating meaningless gestures, our subject appears to follow a goal-directed strategy, in which the imitation goals are represented as relationships between parts of the body and where reproduction of the orientation of the hand takes precedence over reproducing the precise position of contact (see Table 1 ). Such goal-dissociation is in agreement with the goal-directed theory of imitation, in which the imitator decomposes the perceived action into a set of goals according to their relative importance (Bekkering \& Wohlschläger, 2002; Bekkering, Wohlschläger, \& Gattis, 2000; Wohlschläger, Gattis, \& Bekkering, 2003). It also supports the "active intermodal matching" (Meltzoff \& Moore, 1977, 1997) and "body part coding" (Goldenberg et al., 2001) models, in which perceived gestures are encoded as simple relationships between a limited number of defined body parts. Body part coding has the advantage to accommodate novel and meaningless gestures into combinations of familiar elements, hence simplifying recognition and reproduction of the stimuli (Goldenberg, 1996, 1999, 2006; Goldenberg \& Hagmann, 1997; Meltzoff \& Moore, 1997; Sirigu, Grafman, Bressler, \& Sunderland, 1991). In addition, this helps to overcome differences in body shape and size and in perspective between demonstrator and imitator (Goldenberg, 1996, 1999, 2006; Goldenberg \& Hagmann, 1997; Goldenberg et al., 2001).

The observation that the sole reproduction of the position of contact was impaired when information was processed ipsilaterally in the right hemisphere (see Fig. 2A) supports the hypothesis of a left hemisphere dominance for body part coding (Goldenberg et al., 2001). Indeed, whereas coding for the orientation of the hand may be done through extrinsic variables (i.e., according to vertical or horizontal axes), coding for the position of the hand on the face relies on intrinsic variables that pertain to relationships between body parts.

In a previous study, left hemisphere patients showed more impaired imitation of hand vs. finger postures (Goldenberg, 1999). Their deficit was attributed to the greater demands on body part coding for hand postures: the positions of contact between the hand and the head are determined in relation to a multitude of body parts such as the chin, lips, back, or tip of the nose, cheek, or ears. In contrast, finger configurations are composed of a very limited set of uniform elements that differ only in their serial position. Within hand postures, an analogous distinction can be made between position of contact and orientation of the hand. The necessity to consider multiple body parts concerns only the determination of the point of contact between hand and head. The orientation of the hand is generally easier to define because it has less degrees of freedom, and it can be defined in relation to the vertical and horizontal axes of the surrounding space without reference to body parts of the head.

From a computational perspective, the fact that only some of the goals are correctly reproduced at a time may be due to an impaired coordination between two motor programs that individually would achieve each of the goals separately. For example, in our study, one motor program would generate a motion to reach for the specified location on the face with a comfortable canonical hand orientation, whereas the other motor program would generate a motion to satisfy the hand orientation, irrespective of the hand's position in space. To replicate correctly both goals, i.e., specified location and orientation, the two programs must be coordinated. The errors observed in condition LL (see Fig. 4C) may be due to the fact that 
the two imitation goals were not properly coordinated, possibly because part of this coordination must be computed in the left hemisphere. The observation that the patient was able to imitate both goals correctly for some stimuli even when presented in LL may be due to the fact that, in these special cases, the two goals were consistent with the default secondary goal of one of the two motor programs. For instance, this may happen when the motor program to touch the face on a specific location generates a canonical orientation of the hand that is similar to the requested orientation of the hand (such as in stimuli 2 and 4 ). Such impairment in properly coordinating two motor programs may or may not lead to visible errors when the imitation goals are executed sequentially, depending on the order of execution (see Fig. 5). This would explain the differential performance across trials related to one specific stimulus (see Fig. 4C). Importantly, this hypothesis makes one testable prediction: the patient will not make errors in the LL condition when asked to imitate a single imitation goal, i.e., either the orientation of the hand irrespective of the position of contact with the face or to touch the face at a specific location with a "natural" orientation of the hand.

Following evidence for the implication of the left inferior parietal cortex (BA40, near the intraparietal sulcus) in the imitation of meaningless gestures (Decety et al., 1997; Goldenberg \& Karnath, 2006; Haaland et al., 2000; Hermsdörfer et al., 2001; Mühlau et al., 2005; Peigneux et al., 2004; Tessari et al., 2007), we speculate that this brain area may be responsible for the combination of simultaneous movements initiated to satisfy multiple imitation goals, such as to imitate an orientation of the hand while touching the face. In order to satisfy these two imitation goals the brain needs to integrate them into a single movement, a process that requires knowledge about the structure of the body and positions of the body parts. A body of evidence supports the hypothesis that knowledge about body parts relationships is computed in the left intraparietal sulcus. Indeed, the left intraparietal sulcus was activated when subjects were asked to evaluate the distance between body (Corradi-Dell'Acqua, Hesse, Rumiati, \& Fink, 2008), and has also been proposed as the neural substrate of both the "body schema" coding for the dynamic positions of body parts in space (Bonda, Petrides, Frey, \& Evans, 1995; Parsons et al., 1995) and the "body structural description" coding for the spatial relationships among body parts (Felician et al., 2004; Le Clec'H et al., 2000).

The errors observed in the two crossed conditions (RL and LR) were expected, as in these conditions the visual information is processed in a different hemisphere than the motor command, which requires transfer of information through the impaired corpus callosum (see Fig. 2B and C). The majority of errors in these conditions could be linked to a complete or partial reproduction of the previous stimulus and were hence categorized as perseveration errors. These perseveration, two "no-idea" and four "searching" errors suggest that the hemisphere controlling the hand simply did not have access to one or several aspects of the stimulus, and hence the patient executed the immediately preceding stimulus still present at the level of the motor command. Interestingly, we observed very surprising partial perseveration errors where the previous hand orientation was imitated at a correct and updated position of contact (see Fig. 4B), which again speaks in favor of goal-directed imitation.

Finally, we also observed that the errors varied as a function of the stimulus to imitate, such that not all of the stimuli seem to be equal in the face of apraxia. As a general rule, the stimuli where the position of contact with the face was on the palm were easier to imitate than those where the contact position was on the thumb. The difficulty to imitate specific stimuli may be attributed to: (1) the number of constraints defining the gesture, e.g., in some of the stimuli the orientation of the thumb is added as a constraint, (2) how natural the movement is, e.g., is it a movement that one would execute spontaneously in everyday life and (3) how constrained the movement is by the biomechanics of the arm, e.g., the number of possible orientations at the top of the head where the workspace is smaller than in front of the face. Stimuli used for clinical examination of apraxia thus need to be chosen carefully.

\section{Acknowledgements}

This work was funded by the Sport and Rehabilitation Engineering Program at EPFL and the EU Project IST-2004-004370 RobotCub.

\section{Appendix A. Supplementary data}

Supplementary data associated with this article can be found, in the online version, at doi:10.1016/j.neuropsychologia.2010.04.025.

\section{References}

Astafiev, S. V., Stanley, C. M., Shulman, G. L., \& Corbetta, M. (2004). Extrastriate body area in human occipital cortex responds to the performance of motor actions. Nature Neuroscience, 7(5), 542-548.

Bekkering, H., \&Wohlschläger, A. (2002). Action perception and imitation: A tutorial. In W. Prinz, \& B. Hommel (Eds.), Common mechanisms in perception and action. Attention and Performance XIX (pp. 294-314). Oxford: Oxford University Press.

Bekkering, H., Wohlschläger, A., \& Gattis, M. (2000). Imitation of gestures in children is goal-directed. The Quarterly Journal of Experimental Psychology A, 53(1), $153-164$.

Bonda, E., Petrides, M., Frey, S., \& Evans, A. (1995). Neural correlates of mental transformations of the body-in-space. Proceedings of the National Academy of Sciences of the United States of America, 92(24), 11180-11184.

Corradi-Dell'Acqua, C., Hesse, M. D., Rumiati, R. I., \& Fink, G. R. (2008). Where is a nose with respect to a foot? The left posterior parietal cortex processes spatial relationships among body parts. Cerebral Cortex, 18(12), 2879-2890.

De Renzi, E., Faglioni, P., \& Sorgato, P. (1982). Modality-specific and supramodal mechanisms of apraxia. Brain, 105(Pt 2), 301-312.

Decety, J., Grèzes, J., Costes, N., Perani, D., Jeannerod, M., Procyk, E., et al. (1997). Brain activity during observation of actions. Influence of action content and subject's strategy. Brain, 120(Pt 10), 1763-1777.

Downing, P. E., Jiang, Y., Shuman, M., \& Kanwisher, N. (2001). A cortical area selective for visual processing of the human body. Science, 293(5539), 2470-2473.

Felician, O., Romaiguère, P., Anton, J.-L., Nazarian, B., Roth, M., Poncet, M., et al. (2004). The role of human left superior parietal lobule in body part localization. Annals of Neurology, 55(5), 749-751.

Goldenberg, G. (1996). Defective imitation of gestures in patients with damage in the left or right hemispheres. Journal of Neurology, Neurosurgery and Psychiatry, $61,176-180$.

Goldenberg, G. (1999). Matching and imitation of hand and finger postures in patients with damage in the left or right hemispheres. Neuropsychologia, 37(5), 559-566.

Goldenberg, G. (2006). Imitation: Is cognitive neuroscience neglecting apraxia? Trends in Cognitive Sciences, 10(3), 94-95.

Goldenberg, G., \& Hagmann, S. (1997). The meaning of meaningless gestures: A study of visuo-imitative apraxia. Neuropsychologia, 35(3), 333-341.

Goldenberg, G., \& Karnath, H. O. (2006). The neural basis of imitation is body part specific. Journal of Neuroscience, 26(23), 6282-6287.

Goldenberg, G., Laimgruber, K., \& Hermsdörfer, J. (2001). Imitation of gestures by disconnected hemispheres. Neuropsychologia, 39(13), 1432-1443.

Haaland, K. Y., \& Flaherty, D. (1984). The different types of limb apraxia errors made by patients with left vs. right hemisphere damage. Brain and Cognition, 3(4), $370-384$.

Haaland, K. Y., Harrington, D. L., \& Knight, R. T. (2000). Neural representations of skilled movement. Brain, 123(Pt 11), 2306-2313.

Hanna-Pladdy, B., Daniels, S. K., Fieselman, M. A., Thompson, K., Vasterling, J. J., Heilman, K. M., et al. (2001). Praxis lateralization: Errors in right and left hemisphere stroke. Cortex, 37(2), 219-230.

Hanna-Pladdy, B., Heilman, K. M., \& Foundas, A. L. (2001). Cortical and subcortical contributions to ideomotor apraxia. Analysis of task demands and error types. Brain, 124(Pt 12), 2513-2527.

Heilman, K. M., \& Rothi, L. J. G. (1993). Apraxia. In K. M. Heilman, \& E. Valenstein (Eds.), Clinical neuropsychology (3rd ed., pp. 141-163). New York: Oxford University Press.

Hermsdörfer, J., Goldenberg, G., Wachsmuth, C., Conrad, B., Ceballos-Baumann, A. O., Bartenstein, P., et al. (2001). Cortical correlates of gesture processing: Clues to the cerebral mechanisms underlying apraxia during the imitation of meaningless gestures. Neuroimage, 14(1 Pt 1), 149-161.

Hermsdörfer, J., Mai, N., Spatt, J., Marquardt, C., Veltkamp, R., \& Goldenberg, G. (1996). Kinematic analysis of movement imitation in apraxia. Brain, 119(Pt 5), 1575-1586.

Le Clec'H, G., Dehaene, S., Cohen, L., Mehler, J., Dupoux, E., Poline, J. B., et al. (2000). Distinct cortical areas for names of numbers and body parts independent of language and input modality. Neuroimage, 12(4), 381-391. 
Meltzoff, A. N., \& Moore, M. K. (1977). Imitation of facial and manual gestures by human neonates. Science, 198(4312), 75-78.

Meltzoff, A. N., \& Moore, M. K. (1997). Explaining facial imitation: A theoretical model. Early Development and Parenting, 6, 179-192.

Merians, A. S., Clark, M., Poizner, H., Macauley, B., Gonzalez Rothi, L. J., \& Heilman, K. M. (1997). Visual-imitative dissociation apraxia. Neuropsychologia, 35(11), 1483-1490.

Mühlau, M., Hermsdörfer, J., Goldenberg, G., Wohlschläger, A. M., Castrop, F., Stahl, R., et al. (2005). Left inferior parietal dominance in gesture imitation: An fMRI study. Neuropsychologia, 43(7), 1086-1098.

Ochipa, C., Rothi, L. J. G., \& Heilman, K. M. (1994). Conduction apraxia. Journal of Neurology Neurosurgery and Psychiatry, 57(10), 1241-1244.

Parsons, L. M., Fox, P. T., Downs, J. H., Glass, T., Hirsch, T. B., Martin, C. C., et al. (1995). Use of implicit motor imagery for visual shape discrimination as revealed by PET. Nature, 375(6526), 54-58

Peigneux, P., Salmon, E., Van der Linden, M., Garraux, G., Aerts, J., Delfiore, G., et al. (2000). The role of lateral occipitotemporal junction and area MT/V5 in the visual analysis of upper-limb postures. Neuroimage, 11(3), 644-655.

Peigneux, P., Van der Linden, M., Garraux, G., Laureys, S., Degueldre, C., Aerts, J., et al. (2004). Imaging a cognitive model of apraxia: The neural substrate of gesturespecific cognitive processes. Human Brain Mapping, 21(5), 119-142.

Petreska, B., Adriani, M., Blanke, O., \& Billard, A. (2007). Apraxia: A review. Progress in Brain Research, 164, 61-83.
Petreska, B., \& Billard, A. (2006). A neurocomputational model of an imitation deficit following a brain lesion. Lecture Notes in Computer Science, 4131, 770-779.

Petreska, B., \& Billard, A. (2009). Movement curvature planning through force field internal models. Biological Cybernetics, 100(5), 331-350.

Rothi, L. J. G., Ochipa, C., \& Heilman, K. M. (1997). A cognitive neuropsychological model of limb praxis and apraxia. In L. J. G. Rothi, \& K. M. Heilman (Eds.), Apraxia: The neuropsychology of action (pp. 29-49). Hove, UK: Psychology Press.

Rothi, L. J. G., Raymer, A. M., \& Heilman, K. M. (1997). Limb praxis assessment. In L. J. G. Rothi, \& K. M. Heilman (Eds.), Apraxia: The neuropsychology of action (pp. 61-73). Hove, UK: Psychology Press.

Sirigu, A., Grafman, J., Bressler, K., \& Sunderland, T. (1991). Multiple representations contribute to body knowledge processing. Evidence from a case of autotopagnosia. Brain, 114(1), 629-642.

Tessari, A., Canessa, N., Ukmar, M., \& Rumiati, R. I. (2007). Neuropsychological evidence for a strategic control of multiple routes in imitation. Brain, 130(Pt 4), 1111-1126.

Weiss, P. H., Dohle, C., Binkofski, F., Schnitzler, A., Freund, H. J., \& Hefter, H. (2001). Motor impairment in patients with parietal lesions: Disturbances of meaningless arm movement sequences. Neuropsychologia, 39(4), 397-405.

Wohlschläger, A., Gattis, M., \& Bekkering, H. (2003). Action generation and action perception in imitation: An instance of the ideomotor principle. Philosophical Transactions of the Royal Society of London Series B, Biological Sciences, 358(1431), 501-515. 